Proyecciones Journal of Mathematics

Vol. 40, No 6, pp. 1603-1613, December 2021.

Universidad Católica del Norte

Antofagasta - Chile

\title{
A note on convolution operators on Riesz Bounded variation spaces
}

\author{
Lucía Gutierrez \\ Universidad Militar Nueva Granada, Colombia \\ and \\ Oscar M. Guzmán \\ Universidad ECCI, Colombia \\ Received : July 2020. Accepted : May 2021
}

\begin{abstract}
We show some estimates and approximation results of operators of convolution type defined on Riesz Bounded variation spaces in $\mathbf{R}^{n}$. We also state some embedding results that involve the collection of generalized absolutely continuous functions.
\end{abstract}

Keywords: Bounded variation spaces, Riesz Theorem, Convolution operators, Banach algebra.

Subjclass [2010]: 26445 . 


\section{Introduction}

Bounded variation spaces were introduced by C.Jordan in [1] when studied the convergence of Fourier series. Later, F.Riesz introduced in [2] the nowadays known as the Riesz $p$-variation. He showed that $f$ belongs to the Sobolev spaces $W^{1, p}([a . b])$ if an only if

$$
v_{p}(f, I)^{p}=\sup \sum_{k} \frac{\left|f\left(x_{k}\right)-f\left(x_{k-1}\right)\right|^{p}}{\left(x_{k}-x_{k-1}\right)^{p-1}}<\infty,
$$

where the supremun is taken over all the finite partitions $\left\{\left[x_{k-1}, x_{k}\right]\right\}_{k \geq 1}$ of some interval $I$. The latter result is known sometimes as Medelev-Riesz Theorem. Later on, further extensions of this result were given in the one dimensional case. (See for example $[3,4,5]$ ). Also, subsequent works came out in the multidimensional frame (See $[6,7,4]$ ) with a simultaneous interest for understanding the behavior of some integral operators in this scale of functions; maximal operators, operators of convolution type, Mellin integral operators and so on (See $[8,12,10,11])$. In [?] it was introduced the $\varphi$ variation on the multidimensional setting following the approach of Tonelli. Moreover, some approximation results were studied for the operators of convolution type,

$$
T_{w} f(y)=\left(K_{w} * f\right)(y), \quad y \in \mathbf{R}, \quad w>0,
$$

induced by a family of approximation of identities $\left\{K_{w}\right\}_{w>0}$. It turns out, in virtue of the translation invariance nature of classical Lebesgue and Orlicz spaces that further extensions of the result given by eq:RieszTheo facilitate the study of the modulus of continuity defined on bounded variation spaces. In this note, we show some estimates for operators of convolution type defined on the multidimensional Riesz bounded variation spaces as introduced in [7] (See Section 2). In our opinion, this definition captures in a more general fashion the spirit of the classical Riesz bounded variation by means of the oscilation of an arbitrary function $f: \Omega \mapsto R$, which measures the "size" of the image of a given set $E \subseteq \mathbf{R}$ under $f$.

Throughout this paper we use the notation $A B$ and $B A$ to mean that there exist constants $c, C>0$ such that $A \leq c B$ and $B \leq C A$ respectively for some quantities $A, B$. If the former and the later inequalities hold simultaneously we write $A \approx B$. We denote the oscillation of $f$ on a set $E \subseteq \mathbf{R}$ by

$$
\operatorname{osc}_{E} f=\sup _{x \in E} f(x)-\inf _{x \in E} f(x)
$$


Observe that we can change the right part of the definition above by $\sup _{x, y \in E}|f(x)-f(y)|$. We denote the Sobolev space $W_{1}^{p}(\Omega)$ as the collection of functions $f$ such that the weak derivatives $D_{j} f, 1 \leq j \leq n$, belong to the Lebesgue space $L^{p}(\Omega)$.

\section{Preliminaries}

Given $\Omega \subseteq \mathbf{R}$ an open set, let $f: \Omega \mapsto R$ be a measurable function. Following the notation introduced in [7], we say that $f$ has finite Riesz bounded variation on $\Omega$ if

$$
V_{p}(f ; \Omega):=\sup \left(\sum_{B_{k}} \frac{o s c_{B_{k}}(f)^{p}}{r_{k}^{p-n}}\right)^{1 / p}<\infty,
$$

where the supremun is taken over all the disjoint collection of balls $\left\{B_{k}\right\}$ of radii $r_{k}$, contained in $\Omega$. When $V_{p}(f ; \mathbf{R})<\infty$ we denote $V_{p}(f)<\infty$ for the sake of simplicity. Besides, if eq: $\operatorname{RBVpRn}$ holds we write $f \in R B V_{p}(\Omega)$. In the extreme case $p=n$ we denote the class of $n$-Bounded variation function by $B V_{n}(\Omega)$. For further properties in this scale of functions see [13].

Observe that according to eq:RieszTheo and eq:RBVpRn, by considering $\Omega=I$ a bounded open interval we have that

$$
v_{p}(f, I) V_{p}(f, I) .
$$

Hence, the $p$-variation defined in $\mathbf{R}$ by means of the oscillation stands as an extension of the original concept of $p$ variation introduced by Riesz in [2]. Another well know feature in the unidimensional frame is that if $v_{p}(f ; I)<\infty$ then $f$ is a bounded function on $I$. The same phenomenon occurs in the multidimensional case. Without loss of generality, let us assume that $V_{p}(f ; \Omega)<\infty$ for some open bounded set $\Omega$ that contains 0 . Then

$$
\|f\|_{L_{\infty}(\Omega)}|\Omega|^{1-n / p}+|f(0)|<\infty .
$$

However, if $\Omega$ is not bounded $f$ may be not a bounded function. Consider $f(x)=\sqrt{x}, I=(1, \infty)$. Clearly $f$ is not bounded in $I$. On the other hand, by the Riesz-Medvedev Theorem,

$$
v_{p}(f ; I)=\sup _{J \subseteq I}\left\|f^{\prime}\right\|_{L_{p}}<\infty, \quad p>2 .
$$

where the supremum is taken over all the bounded intervals $J$ contained in I. Not long ago, Barza and Lind stated a multidimensional Riesz-Medvedev 
type Theorem. This result gives a simple variational characterization of the Sobolev spaces $W_{1}^{p}(\Omega)$.

Lemma 2.1 (Barza-Lind, [7]). Given $\Omega \subseteq \mathbf{R}$, let $p>n$. Then $f \in$ $W_{p}^{1}(\Omega)$ if and only if $f$ can be modified in a set of measure 0 to be continuous and $V_{p}(f ; \Omega)<\infty$. Moreover

$$
\nabla f_{L^{p}(\Omega)} \approx V_{p}(f ; \Omega) .
$$

From Lemma 2.1, if $\Omega$ is a bounded open set we have that for $1 \leq p \leq$ $q<\infty$

$$
R B V_{q}(\Omega) \hookrightarrow R B V_{p}(\Omega) .
$$

This is also a straightforward consequence of the Hol̂der inequality. A well known embedding in the unidimensional frame is $R B V_{p}([a, b]) \subset$ $A C([a, b]), p>1$. We recall an equivalent definition of absolute continuity in the multidimensional setting (see [13]). We say that a function $f: \Omega \mapsto R$ is $n$-absolutely continuous in $\Omega\left(A C^{n}(\Omega)\right)$ if for any $\delta>0$ there exists $\varepsilon>0$ such that for every finite disjoint collection of closed balls $\left\{B_{k}\right\}$ contained in $\Omega$ we have

$$
\sum_{B_{k}}\left(\operatorname{osc}_{B_{k}} f\right)^{n}<\varepsilon \quad \text { whenever } \quad \sum_{B_{k}}\left|B_{k}\right|<\delta .
$$

Proposition 2.2. $\Omega$ be an open set and $p>n$. Then

$$
R B V_{p}(\Omega) \subseteq A C^{n}(\Omega) .
$$

Proof. Given $\varepsilon>0$, choose $\delta<\left(\varepsilon / V_{p}(f ; \Omega)^{p / n}\right)^{n / s}$. We set a finite disjoint family of balls $\left\{B_{k}\right\}$ contained in $\Omega$ and $f \in R B V_{p}(\Omega)$. Consider $s>1$ such that $1 / p+1 / s=1 / n$. By the Hol̂der inequality we obtain

$$
\begin{aligned}
\sum_{B_{k}}\left(\operatorname{osc}_{B_{k}} f\right)^{n} & \leq\left(\sum_{B_{k}} \frac{\left(\operatorname{osc}_{B_{k}} f\right)^{p}}{r_{k}^{p-n}}\right)^{p / n}\left(\sum_{B_{k}} r_{k}^{n}\right)^{s / n} \\
& V_{p}(f ; \Omega)^{p / n}\left(\sum_{B_{k}}\left|B_{k}\right|\right)^{s / n}<\varepsilon .
\end{aligned}
$$

Since $\varepsilon>0$ is arbitrary, we conclude $f \in A C^{n}(\Omega)$.

Observe that in the extreme value $p=n, R B V_{p}(\Omega)$ coincides with the bounded variation spaces $B V^{n}(\Omega)$ introduced in [13]. Furthermore, the following embedding also holds

Proposition 2.3. Let $f$ be a Lipschitz function in $\Omega,|\Omega|<\infty$. Then $f \in R B V_{p}(\Omega)$. 
Proof. Let $B$ a ball of radius $r_{B}$ contained in $\Omega$, and consider $x, y \in B$, then $|f(x)-f(y)| \leq C|x-y|$ implies

$$
\operatorname{osc}_{B} f r_{B},
$$

So, for a fixed collection $\left\{B_{k}\right\}$ of balls contained in $\Omega$ we have

$$
\sum_{B_{k}}\left(\frac{\operatorname{osc}_{B_{k}} f}{r_{k}}\right)^{p} r_{k}^{n} \sum_{B_{k}}\left|B_{k}\right| \leq|\Omega|<\infty
$$

. Hence $V_{p}(f ; \Omega)<\infty$.

Since $\operatorname{osc}_{E}(f+g) \leq \operatorname{osc}_{E}(f)+\operatorname{osc}_{E}(g), E \subseteq \mathbf{R}$, then $V_{p}(f+g ; \Omega) V_{p}(f ; \Omega)+$ $V_{p}(g ; \Omega)$. Besides, $V_{p}(f ; \Omega)=0$ if and only if $f$ is a constant function for all $x \in \Omega$. Therefore $V_{p}(\cdot ; \Omega)$ is not a norm in $R B V_{p}(\Omega)$. However, we can endow $R B V_{p}(\Omega)$ with a norm. The following result shows that $R B V_{p}$ is closed under the multiplication of functions.

Lemma 2.4. Let $f, g \in R B V_{p}(\Omega)$. Then $f g \in R B V_{p}(\Omega)$.

Proof. Set a disjoint collection of balls $\left\{B_{k}\right\}$ contained in $\Omega$, since $f, g$ are bounded functions in $\Omega$, we estimate as follows

so

$$
\begin{aligned}
\sum_{B_{k}}\left(\frac{o s c_{B_{k}}(f g)}{r_{k}}\right)^{p} r_{k}^{n} & =\sum_{B_{k}}\left(\frac{\sup _{x, y \in B_{k}}|(f g)(x)-(f g)(y)|}{r_{k}}\right)^{p} r_{k}^{n} \\
& \leq \sum_{B_{k}}\left(\frac{\left|\|g\|_{L_{\infty}(\Omega)} o s c_{B_{k}} f+\|f\|_{L_{\infty}(\Omega)} o s c_{B_{k}} g\right|}{r_{k}}\right)^{p} r_{k}^{n} \\
& \|g\|_{L_{\infty}(\Omega)}^{p} \sum_{B_{k}}\left(\frac{o s c_{B_{k}} f}{r_{k}}\right)^{p} r_{k}^{n}+\|f\|_{L_{\infty}(\Omega)}^{p} \sum_{B_{k}}\left(\frac{o s c_{B_{k}} g}{r_{k}}\right)^{p} r_{k}^{n} \\
& \|g\|_{L_{\infty}(\Omega)}^{p} V_{p}(f ; \Omega)^{p}+\|f\|_{L_{\infty}(\Omega)}^{p} V_{p}(g ; \Omega)^{p},
\end{aligned}
$$

$$
V_{p}(f g ; \Omega)\|g\|_{L_{\infty}(\Omega)} V_{p}(f ; \Omega)+\|f\|_{L_{\infty}(\Omega)} V_{p}(g ; \Omega) .
$$

Observe that an alternative proof can be performed by Lemma 2.1, the product rule for weak derivatives and the Minkownski inequality.

Proposition 2.5. Assume $\Omega$ is an open set and $1<p<\infty$. Then the functional defined by

$$
\cdot R B V_{p}(\Omega)=\|\cdot\|_{L_{\infty}(\Omega)}+V_{p}(\cdot ; \Omega)
$$

is a norm in $R B V_{p}(\Omega)$. Moreover, $R B V_{p}(\Omega)$ is a Banach algebra. 
Proof. Clearly $\cdot_{R B V_{p}(\Omega)}$ is a norm. Let $f, g \in R B V_{p}(\Omega)$, by Lemma 2.4 we have

$$
\begin{aligned}
f g_{R B V_{p}(\Omega)} & \|f\|_{L_{\infty}}\|g\|_{L_{\infty}}+\|g\|_{L_{\infty}} V_{p}(f ; \Omega)+\|f\|_{L_{\infty}} V_{p}(g ; \Omega) \\
& \leq\left(\|f\|_{L_{\infty}}+V_{p}(f ; \Omega)\right)\left(\|g\|_{L_{\infty}}+V_{p}(g ; \Omega)\right)=f_{R B V_{p}(\Omega)} g_{R B V_{p}(\Omega)}
\end{aligned}
$$

Remark 2.6. Assume that $p>n$ and set $\|\cdot\|_{R B V_{p}(\Omega)}=\|\cdot\|_{L_{p}(\Omega)}+V_{p}(\cdot ; \Omega)$. By virtue of the Morrey inequality (see [14], Theorem 9.12) and Lemma 2.1 , the norms $\cdot R B V_{p}(\Omega)$ and $\|\cdot\|_{R B V_{p}(\Omega)}$ are equivalent.

The next result is a Fatou type Lemma for $V_{p}(\cdot ; \Omega)$.

Proposition 2.7. Given $\Omega$ an open set, let $\left\{f_{k}\right\}$ be a sequence of bounded functions in $\Omega$. Assume that $f_{k}(x) \rightarrow f(x), k \rightarrow \infty$, for every $x \in \Omega$, . Then

$$
V_{p}(f ; \Omega) \leq \liminf _{k \rightarrow \infty} V_{p}\left(f_{k} ; \Omega\right)
$$

Proof. Fix a collection of disjoint balls $\mathcal{D}=\{B\}$ contained in $\Omega$. Then

$$
V_{p}\left(f_{k} ; \mathcal{D}\right)=\sum_{B \in \mathcal{D}}\left(\frac{\operatorname{osc}_{B}\left(f_{k}\right)}{r_{B}}\right)^{p} r^{n} \leq V_{p}\left(f_{k}\right)
$$

On the other hand, since $\left\{f_{k}\right\}$ is a bounded sequence in every $B \in \mathcal{D}$ and because the continuity of $t \mapsto t^{p}, p>1$, we obtain

$$
\left|V_{p}(f ; \mathcal{D})-V_{p}\left(f_{k} ; \mathcal{D}\right)\right| \leq \sum_{B \in \mathcal{D}}\left|\operatorname{osc}_{B}\left(f_{k}\right)^{p}-\operatorname{osc}_{B}(f)^{p}\right| \frac{1}{r_{B}^{p-n}} \rightarrow 0,
$$

when $k \rightarrow \infty$. So,

$$
V_{p}(f ; \mathcal{D}) \leq \liminf _{k \rightarrow \infty} V_{p}\left(f_{k} ; \Omega\right),
$$

for every collection $\mathcal{D}$. Then the result follows.

\section{Convolution Operators on $R B V_{p}(\mathbf{R})$}

In this section we prove some approximation results of Integral operators in $R B V_{p}(\mathbf{R})$ modular. We recall the definition of approximate identities.

Definition 3.1. A family of functions $\left\{K_{w}\right\}_{w>0}$ is said to be approximate identities if:

(i) $K_{w} \in L^{1}(\mathbf{R})$ for any $w>0$ and $M=\sup _{w>0} K_{w 1}<\infty$. 
(ii) For every $w>0$,

$$
\int_{\mathbf{R}} K_{w}(x) x=1 .
$$

(iii) For every $\delta>0$,

$$
\lim _{w \rightarrow \infty} \int_{x \geq \delta} K_{w}(x) x=0 .
$$

Let us consider the operator $T_{w}$ given by

$$
\left(T_{w} f\right)(x)=\int_{\mathbf{R}} K_{w}(x-y) f(y) y,
$$

which is known as the family of convolution operators associated to $\left\{K_{w}\right\}_{w>0}$. We point out that $T_{w}$ is well defined some scale of functions, for example $L^{p}(\mathbf{R})$ and $C_{0}(\mathbf{R})$. We assume in the next results that $f \in R B V_{p}(\mathbf{R})$ endowed with the norm $\|\cdot\|_{R B V_{p}}$.

Proposition 3.2. Let $T_{w}$ be an operator that satisfies ( $i$ ) and (ii) above. If $f \in R B V_{p}(\mathbf{R})$, then

$$
T_{w}: R B V_{p}(\mathbf{R}) \hookrightarrow R B V_{p}(\mathbf{R}) .
$$

Proof. We show that $V_{p}\left(T_{w} f\right) V_{p}(f)$. Fix a disjoint collection of balls $\mathcal{F}=\left\{B_{k}\right\}$ of radius $r_{k}$. By the Definition 3.1 and by the Jensen inequality we estimate as follows

$$
\begin{aligned}
\sigma_{p}\left(T_{w} f ; \mathcal{F}\right) & =\sum_{B_{k}}\left(\frac{o s c_{B_{k}}\left(T_{w} f\right)}{r_{k}}\right)^{p} r_{k}^{n}=\sum_{B_{k}}\left(\frac{\sup _{t, z \in B_{k}}\left|T_{w} f(t)-T_{w} f(z)\right|}{r_{k}}\right)^{p} r_{k}^{n} \\
& =\sum_{B_{k}}\left(\frac{\sup _{t, z \in B_{k}} \int_{\mathbf{R}} K_{w}(y)(f(t-y)-f(z-y)) y}{r_{k}}\right)^{p} r_{k}^{p} \\
& \leq \sum_{B_{k}}\left(\frac{M \int_{\mathbf{R}}\left|K_{w}(y)\right| o s c_{B_{k}} f(\cdot-y) y}{r_{k} \int_{\mathbf{R}}\left|K_{w}(y)\right| y}\right)^{p} r_{k}^{n} \\
& \frac{\int_{\mathbf{R}}\left|K_{w}(y)\right| \sum_{B_{k}}\left(\frac{o s c_{B_{k}} f(-y)}{r_{k}}\right)^{p} r_{k}^{n} y}{\int_{\mathbf{R}}\left|K_{w}(y)\right| y} V_{p}(f ; \mathbf{R})<\infty,
\end{aligned}
$$

which ends the proof.

A function $f$ is said to be Lipchitz continuous at the point $x$ if its upper pointwise Lipchitz constant at $x$ is finite, i.e.

$$
L_{f}(x):=\limsup _{y \rightarrow x} \frac{|f(x)-f(y)|}{|x-y|}<\infty
$$


Evidently, if $f \in \operatorname{Lip}(\Omega)$ then $f$ is a Lipchitz continuous function. On the other hand, when $\Omega$ is a quasiconvex set every Lipchitz continuous function is also a Lipchitz function (See [15], Lemma 2.2). As a consequence of the Vitalli covering Lemma, $L_{f}$ satisfies the weak estimate

$$
\left|\left\{x \in \mathbf{R}: L_{f}(x)>t\right\}\right|\left(\frac{V_{p}(f)}{t}\right)^{p}, \quad t>0, \quad p>n .
$$

cf. [7].

Corollary 3.3. Let $f \in R B V_{p}(\mathbf{R})$ and $p>n$. Then $T_{w}$ is Lipchitz continuous almost everywhere.

Proof. In fact, the result $\left|\mathbf{R} \backslash\left\{x \in \mathbf{R}: L_{\left(T_{w} f\right)}(x)<\infty\right\}\right|=0$ follows from the weak type inequality,

$$
\left|\left\{x \in \mathbf{R}: L_{\left(T_{w} f\right)}(x)>t\right\}\right|\left(\frac{V_{p}(f)}{t}\right)^{p},
$$

which is interesting in its own right. The inequality (3.3) is immediate taking into account Proposition 3.2 and (3.2).

Given $f \in R B V_{p}(\mathbf{R})$, the $V_{p}$-modulus of continuity of order $\alpha, \delta>0$, is given by

$$
\omega_{p}(f, \delta):=\sup _{|y| \leq \delta} V_{p}\left(\tau_{y} f-f\right),
$$

where $\left(\tau_{y} f\right)(x)=f(x-y)$ and $x, y \in \mathbf{R}$.

Proposition 3.4. Let $f \in R B V_{p}(\mathbf{R})$. Then

$$
\lim _{\delta \rightarrow 0^{+}} \omega_{p}(f, \delta)=0
$$

Proof. $\quad$ By Lemma 2.1, $D_{j} f \in L_{p}(\mathbf{R}), j=1, \cdots, n$. Since $L_{p}(\mathbf{R})$ is translation invariant we have

$$
V_{p}\left(\tau_{y} f-f\right)^{p} \sum_{j=1}^{n} \int_{\mathbf{R}}\left|\tau_{y} D_{j} f(x)-D_{j} f(x)\right|^{p} x \rightarrow 0,
$$

when $|y| \rightarrow 0$. So, the result follows.

The next result states the continuity of $T_{w}$ under the $p$-variation. 
THEOREM 3.5. Given $f \in R B V_{p}(\mathbf{R})$, let $\left\{K_{w}\right\}_{w>0}$ be a sequence of aproximation of identities. Then

$$
\lim _{w \rightarrow \infty} V_{p}\left(T_{w} f-f\right)=0 .
$$

Proof. Let $\mathcal{F}$ a fixed disjoint collection of balls. By the generalized Jensen inequality we have

$$
\begin{aligned}
\sigma_{p}\left(T_{w} f-f ; \mathcal{F}\right)^{p} & =\sum_{B_{k}}\left(\frac{\operatorname{osc}_{B_{k}}\left(\int_{\mathbf{R}} K_{w}(y)[f(\cdot-y)-f(\cdot)] y\right)}{r_{k}}\right)^{p} r_{k}^{n} \\
& \leq \sum_{B_{k}}\left(\frac{M \int_{\mathbf{R}}\left|K_{w}(y)\right| o s c_{B_{k}}(f(\cdot-y)-f(\cdot)) y}{r_{k} \int_{\mathbf{R}}\left|K_{w}(y)\right| y}\right)^{p} r_{k}^{n} \\
& \sum_{B_{k}} \frac{\int_{\mathbf{R}}\left|K_{w}(y)\right| o s c_{B_{k}}^{p}(f(\cdot-y)-f(\cdot) y}{r_{k}^{p-n} \int_{\mathbf{R}}\left|K_{w}(y)\right| y} \\
& \int_{|y|>\delta}\left|K_{w}(y)\right| \sum_{B_{k}}\left(\frac{o s c_{B_{k}}(f(\cdot-y)-f(\cdot))}{r_{k}}\right)^{p} r_{k}^{n} y \\
& +\int_{|y| \leq \delta}\left|K_{w}(y)\right| \sum_{B_{k}}\left(\frac{o s c_{B_{k}}(f(\cdot-y)-f(\cdot))}{r_{k}}\right)^{p} r_{k}^{n} y=I+I I .
\end{aligned}
$$

By Lemma 2.1 and by the Minkowski inequality we obtain

$$
\begin{aligned}
I \leq & \int_{|y|>\delta}\left|K_{w}(y)\right| V_{p}^{p}(f(\cdot-y)-f(\cdot)) y \\
& \int_{|y|>\delta}\left|K_{w}(y)\right| \nabla(f(\cdot-y)-f(\cdot))_{L p}^{p} y \\
& \int_{|y|>\delta}\left|K_{w}(y)\right| \nabla f_{L_{p}}^{p} y .
\end{aligned}
$$

We estimate $I I$ as follows

$$
\text { II } \int_{|y| \leq \delta}\left|K_{w}(y)\right| V_{p}^{p}(f(\cdot-y)-f(\cdot)) y \quad \omega_{p}(f, \delta)^{p} \int_{|y| \leq \delta}\left|K_{w}(y)\right| y .
$$

from the previous estimates it follows

$$
V_{p}\left(T_{w} f-f, \mathbf{R}\right)^{p} \nabla f_{L_{p}}^{p} \int_{|y|>\delta}\left|K_{w}(y)\right| y+\omega_{p}(f, \delta)^{p} \int_{|y| \leq \delta}\left|K_{w}(y)\right| y,
$$

due to Proposition 3.4 and eq:Aprox-Def3 the theorem follows.

\section{Acknowledgments}

This paper is a product of the research project cias 2944 funded and supported by the "Universidad Militar Nueva Granada", sub direction of Investigation dependency. 


\section{References}

[1] C. Jordan, "Sur la series de Fourier", Comptes Rendus de l'Académie des Sciences Paris, vol. 92, pp. 228-230, 1881.

[2] F. Riesz, "Untersuchungen uber systeme integrierbarer funktrionen", Mathematische annales, vol. 69, no. 4, pp. 449-497, 1910.

[3] J. Appell, J. Banas, and N. Merentes, Bounded variation and around. Berlin: De Gruyter, 2014

[4] L. Tonelli, "Su alcuni concetti dellánalisi moderna”, Annali della Scuola Normale Superiore di Pisa, Classe di Scienze, vol. 11, no. 1-2, pp. 107-118, 1942.

[5] L. Young, "Sur une généralisation de la notion de variation de puissance p-ieme bornee au sens de M. Wiener, et sur la convergence des séries de Fourier", Comptes Rendus de l'Académie des Sciences Paris, vol. 204, pp. 470-472, 1937.

[6] B. Lenze, "On constructive one-sided approximation of multivariate functions of bounded variation", Numerical functional analysis and optimization, vol. 11, no.1-2, pp. 55-83, 1990.

[7] S. Barza and M. Lind, "A new variational characterization of Sobolev spaces", The journal of geometric analysis, vol. 25, no. 4, pp. 2185-2195, 2015.

[8] L. Angeoloni, "A new concept of multidimensional variation in the sense of Riesz and applications to integral operators", Mediterranean journal of mathematics, vol. 14, no. 4, Art. ID. 149, 2017.

[9] L. Angeloni and G. Vinti, "A review on approximation results for integral operators in the space of functions of bounded variation", Journal of function spaces, vol. 2016, Art. ID. 3843921.

[10] C. Bardaro, J. Musielak, and G. Vinti, Nonlinear integral operators and applications. Berlin: De Gruyter, 2003.

[11] P. Butzer and S. Jansche, "A direct approach to the Mellin transform", Journal of Fourier analysis and applications, vol. 3, no. 4, pp. 325-376, 1997. 
[12] L. Angeloni and G. Vinti, "Convergence andd rate of approximation for linear integral operators in $\mathrm{BV}^{\phi}$-spaces in multidimensional settins", Journal of mathematical analysis and applications, vol. 349, no. 2, pp. 317-334, 2009.

[13] J. Maly, "Absolutely continuous functions of several variables", Journal of mathematical analysis and applications, vol. 231, no. 2, pp. 492-508, 1999.

[14] H. Brezis, Functional analysis, Sobolev spaces and partial differential equations. New York, NY: Springer, 2011.

[15] J. Heinonen, "Lectures on Lipschitz analysis", University of Jyväskylä, Department of Mathematics and Statistics, Finland, Rep., 100, 2005.

\section{Lucía Gutierrez}

Departamento de Matemáticas, Universidad Militar Nueva Granada,

Colombia,

Bogotá

e-mail: lucia.gutierrez@unimilitar.edu.co

and

Oscar M. Guzmán

Dirección de Ciencias Básicas, Universidad ECCI,

Colombia

Bogotá

e-mail: oguzmanf@ecci.edu.co 